\title{
DEMOGRAPHIC, CLINICAL AND MAMMOGRAPHIC CHARACTERISTICS OF INVASIVE DUCTAL CARCINOMA OF THE BREAST: A SRI LANKAN EXPERIENCE
}

\author{
Perera DC ${ }^{1}$, Hettiarachchi GB ${ }^{1}$, Ratnatunga $\mathrm{NVI}^{2}$, Kumarasiri $\mathrm{PVR}^{3}$, Hewavithana $\mathrm{PB}^{1}$ \\ ${ }^{1}$ Department of Radiology, Faculty of Medicine, University of Peradeniya, Sri Lanka \\ ${ }^{2}$ Department of Pathology, Faculty of Medicine, University of Peradeniya, Sri Lanka \\ ${ }^{3}$ Department of Community Medicine, Faculty of Medicine, University of Peradeniya, \\ Sri Lanka
}

DOI: http://doi.org/10.4038/sljr.v2i2.48

\begin{abstract}
Breast cancer (BC) ranks the highest among all cancers of women worldwide. Mammography is the most widely utilized imaging tool for evaluation of breast cancer with the final diagnosis being made on histopathology. This study aimed at describing the demographic, clinical and mammographic characteristics of histologically proven invasive ductal carcinoma (IDC) of breast in a group of Sri Lankan women. The study was carried out using a database on mammography maintained by the principal investigator. Study sample consisted of 177 subjects. The mean age of subjects was 52.2 years $(\mathrm{SD} \pm 1.1$ ). Majority (63.8\%) were postmenopausalwomen. $93 \%$ of them presented with symptomatic breast disease, and the commonest symptom was a palpable mass $(90.7 \%)$. Presentation for mammography after observing symptoms showed a median delay of 28 days. BC was found mostly in involuting type of breasts. Commonest mammography characteristic was a mass (86.4\%). Size of the mass was between $2 \mathrm{~cm}$ to $5 \mathrm{~cm}$ in majority (84.3\%) with T stage II disease. In conclusion, mean age of the subjects was comparable to other Asian countries but relatively lower than that of the west. Majority of patients presented with a palpable mass within four weeks from the onset of symptoms. The size of the mass did not show a significant correlation with the duration of symptoms and the age. This study did not find a significant association between mammographic breast density with IDC.
\end{abstract}

Keywords:Breast cancer,Invasive ductal carcinoma, Mammography

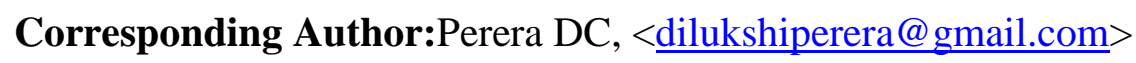

(iD) http://orcid.org/0000-0003-1179-6131

CC) (1) This is an open-access article distributed under the terms of the Creative Commons Attribution License, which permits unrestricted use distribution, and reproduction in any medium, provided the original author and source are credited. 


\section{Introduction}

There is a significant increase in the incidence of breast cancer (BC) worldwide with 1 million new cases each year ${ }^{1}$. BC contributes to $18 \%$ of all cancers of women ${ }^{1}$.The age standardised incidence rates (ASR) of BC vary from 19.3 in Eastern Africa to 89.9 in Western Europe $^{2}$. In Sri Lanka too, breast cancer ranks highest among all the cancers in female and ASR of BC has risen substantially from 9.4 in 1985 to 23 in $2010^{3}$. Age is one of the most important risk factors of developing a $\mathrm{BC}$. At the age of 30 a woman has $0.44 \%$ of risk of developing $\mathrm{BC}$ over a period of 10 years where as at the age of 60 years it increases to $3.46 \%{ }^{4}$. According to the cancer registry 2010 in Sri Lanka, higher proportion of BC were in above 50 years age groups and it was highest in the age category of 6064years ${ }^{3}$. It has been shown that there is a higher risk of breast cancer after menopause, but the data on incidence of $\mathrm{BC}$ during the pre and postmenopausal years of women has been controversial $^{5,6}$.

A palpable mass is the most common symptom and the initial complaint for about $50 \%$ of the breast cancer patients ${ }^{7}$. The overall sensitivity of symptoms in detecting $\mathrm{BC}$ is found to be $35.5 \%^{8}$.

There are many imaging methods available to detect $\mathrm{BC}$ such as ultrasonography, MRI and scinti-mammography ( $\mathrm{Tc}^{99 \mathrm{~m}}$ Sestamibi) with variable degrees of sensitivity, but mammography remains the most widely used method up to date, with a sensitivity ranging from $75 \%$ to $90 \%$ and specificity from $90 \%$ to $95 \%$.

Mammographic breast density is a known strong predictor of breast cancer ${ }^{10,11}$. Wolfe proposed a classification of breast densities on mammography and he defined four parenchymal patterns also known as Wolfe grades along with the risk of breast cancer related to each category in 1976. They are: N1 pattern of fatty radiolucent breast, P1 pattern with less than 25\% prominent fibroglandular tissue (FG), P2 pattern with more than $25 \%$ prominent $\mathrm{FG}$ tissue and DY pattern with dense FG tissue ${ }^{12}$.According to Wolfe's study, N1 pattern has a $0.1 \%$ incidence of breast cancer compared to P2 and DY patterns where there is 17 to 22 -fold increase in the incidence of $\mathrm{BC}$ respectively ${ }^{12}$.

It has been reported that certain histological types of $\mathrm{BC}$ have a tendency to show a specific mammographic appearance but there are results on the contrary ${ }^{13,14,15}$.In high-grade ductal carcinoma in situ (DCIS), debris from tumour necrosis leads to characteristic pleomorphic microcalcification, which gives a branching pattern on mammography. Invasive ductal carcinoma (IDC) induces fibrous response when it infiltrates outside the duct and it contributes to mass formation along with the malignant cells ${ }^{16}$. This explains the variable appearances on mammography ranging from visible mass particularly with an irregular or spiculated margin, architectural distortion, asymmetric density and pleomorphic microcalcification. Further invasion of the IDC leads to skin thickening, nipple 
retraction, spread to locoregional lymph nodes and distant metastases.

Moreover, breast cancer management strategyis another important fact considered in the interpretation of mammogram. The most widely used such guideline is the Breast Imaging Reporting and Data System (BIRADS) categories ${ }^{17}$.

The aim of this study was to describe the clinical presentation, demographics and mammographic characteristics in a group of Sri Lankan female population, who were confirmed to have IDC on histopathology.

\section{Materials and Methods}

This was a descriptive study, carried out using a mammography database maintained by the principal investigator at a mammography facility between 2006 and 2016 in Kandy district, Sri Lanka. Inclusion criterion was the subjects with $\mathrm{BC}$ proven on histopathology as IDC. Among 209 BC cases in the database, 177 with IDC were selected as subjects.Mammograms were carried out by using a Bennett USA mammography unit by two experienced radiographers. Data on demographic and mammographic characteristics were recorded in a pre-coded questionnaire. Mammographic characteristics namely, size, density, margin and site of the mass, presence of microcalcification, architectural distortion, skin thickening, nipple retraction and asymmetric density on standard views (MLO and CC), reported by the principal investigator with more than 15 years experience on imaging $\mathrm{BC}$ were considered in this study.Double reading of each mammogram was performed by the same investigator. Histopathological reporting was done by an experienced senior histopathologist. Standard statistical methods such as chi-square test, Spearman correlation and frequency were used. Statistical Package for the Social Sciences statistical software (version 20.0; SPSS Inc., Chicago, IL, USA) was used in analysis. Ethical clearance was obtained from the ethics review committee of the Faculty of Medicine, University of Peradeniya.

\section{Results}

Out of $84.7 \%$ IDC subjects, $80.4 \%$ were in isolation whereas $4.3 \%$ had coexistent DCIS (Table 1). Age ranged from 30 to 83 years (mean age 52.2, SD \pm 1.1 ). There were $32.2 \% \quad(n=57) \quad$ and $31.1 \% \quad(n=55)$ representing age groups 40-49 and 50-59 years. $10.7 \% \quad(n=19), 26 \% \quad(n=46)$ were below 40 years and above 60 years respectively. $63.8 \%(n=111)$ of them were postmenopausal where as $36.2 \% \quad(n=63)$ were premenopausal and the mean age of menopause was $48.1(\mathrm{SD} \pm 5.1)$ (Table 1$)$.

Of the total study population, 93\% $(n=161)$ presented with symptomatic breast disease.The most common clinical presentation of the symptomatic women was a palpable mass $(90.7 \%, n=157) .63 \%$ $(n=109)$ of the masses were isolated and $27.7 \% \quad(n=48)$ were associated with few other symptoms such as mastalgia $(25.4 \%)$, nipple discharge $(2.9 \%)$ or skin dimpling $(2.3 \%)$. Second commonest cause of presentation for mammography was mastalgia, mostly with another symptom $(25.4 \%)$ than in isolation (2.3\%). Seven percent $\quad(n=12) \quad$ women sought 
mammography without any symptoms. With regard to the time taken for presentation for mammography from the point of symptoms, delay of one week was found in $15.4 \%$ $(n=23)$ women and more than three months in $21.5 \%$, but $63.1 \%(\mathrm{n}=94)$ had presented within one to eleven weeks after observing symptoms with a median delay of 28 days (Table 1).

Among them, 70.5\% $(\mathrm{n}=120)$ had involuting P1 Wolfe type of breast on mammography whereas $21.8 \%(n=37)$ had adipose breasts and only $2.9 \%(n=5), 4.7 \%(n=8)$ had dense and involuting $\mathrm{P} 2$ types respectively (Table 2). Among the premenopausal group $73.8 \%$ $(n=45)$ and $68.8 \% \quad(n=75)$ of the postmenopausal group had P1 type of involuting breasts. Pre and postmenopausal groups had $4.9 \%$ and $1.8 \%$ DY type and $11.5 \%$ and $0.9 \%$ P2 types respectively (Table 2).

When considering the mammographic features of IDC among subjects, 86.4\% $(\mathrm{n}=153)$ had a mass on mammograms. $43.8 \% \quad(n=67)$ were in isolation without microcalcification, nipple retraction, skin thickening or architectural distortion (Table 3). On analysis of the side and the site of the mass lesion on mammogram, 59\% $(n=66)$ were found in right breast and according to quadrant, right upper outer quadrant (RUOQ) had $43.8 \%(n=49)$, left upper outer quadrant (LUOQ), left upper inner quadrant (LUIQ), and right upper inner quadrant (RUIQ) had 23.2\%, 9.8\% and $8.0 \%$ respectively (Table 3). Unilateral disease was found in $98.6 \%(n=140)$ of the subjects. With regard to the focal distribution of the masses, $91.3 \% \quad(n=126)$ were found to be unifocal. $5.8 \%(n=8)$ and $2.9 \%(n=4)$ of them had multifocal and multicentric disease respectively (Table 3). Further analysis of the characteristics of the mass on mammograms revealed that $67.4 \%(n=93)$ of the masses were of high-density while $26.8 \%(\mathrm{n}=37)$ were isodense (Table 3$)$. There were $87 \% \quad(n=127)$ masses with a spiculated margin and $84.3 \%(n=129)$ of the masses were between $2-5 \mathrm{~cm}$ in size (mean $3.1 \pm$ SD1.5) of T stage II. Only $5.2 \%(n=8)$ had more than $5 \mathrm{~cm}$ masses (T stage III) and $10.5 \%(n=16)$ had less than $2 \mathrm{~cm}$ masses ( $\mathrm{T}$ stage I) on mammography (Table 3 ).

$27.6 \% \quad(n=49)$ of the subjects showed microcalcification on the mammogram. Among them, 46 subjects presented with a mass combined with other mammographic features, three subjects showed microcalcification with other features but without a mass (Table 3). 84.2\% had architectural distortion either in isolation $(6.2 \%)$ or with other mammographic signs. $78.4 \%(n=124)$ of the subjects were in the BIRADS 5 category whereas $5.1 \%(n=8)$ in each BIRADS 3 and BIRADS 6 categories. Analysis of the correlation between the duration of symptoms and the tumour size did not reveal any significant association (Table 4).Similarly age of the patient and tumour size also did not show any significant association (Table 5). Of the 177 BC patients who underwent mammographic examination, 51 had metastatic axillary lymph nodes (LN). However, mammography detected only 21 (41.2\%) metastatic LN (sensitivity=41.2\%) (Table6). 


\section{Discussion}

Breast cancer is a complex disease of many histological and molecular subtypes with a wide spectrum of clinical presentations, risk factors and outcomes. In the current management of $\mathrm{BC}$, mammography plays a crucial role in establishing the diagnosis.Randomized clinical trials have shown that mammography has contributed to the reduction of overall breast cancer mortality by $30 \%$ in women aged 50 to 59 years who were screened for $\mathrm{BC}$ on mammography ${ }^{9}$. Demographic, clinical and mammographic characteristics on various histological types of $\mathrm{BC}$ are well documented in the literature, based on western data, however, revisits in local literature is sparse. Invasive ductal carcinoma was exclusively selected in this study, as this is the commonest $(84.7 \%)$ among all the histological types of this study population. This is comparable with global figures of 70\%-90\% IDC among all histological types ${ }^{18,19}$.

It revealed that the majority of women with IDC in the age ranges between 40-49 (32.2\%) and 50-59 (31.1\%) years with a mean age of 52.2( $\mathrm{SD} \pm 1.1)$ years. This age distribution is comparable with the figures given for Asia, but it is somewhat lower than that of western countries where the mean age is between $60-70$ years ${ }^{5,20,21}$. However, age specific $\mathrm{BC}$ incidence showed a peak incidence at 60-64 years in all types of $\mathrm{BC}^{3}$. Incidence of $\mathrm{BC}$ among pre and postmenopausal years of women has been extensively investigated in various studies, but the results are inconclusive ${ }^{5,6}$. Studies done in Spain and Canada showed that IDC was more common in postmenopausal than premenopausal women ${ }^{13,15}$. However, there were results on the contrary in a study by Jiang et al. who found that $57.4 \%$ were premenopausal ${ }^{5}$. Moreover, Lancet oncology 2012, based on a meta-analysis reported that premenopausal woman have a greater risk of breast cancer than postmenopausal women of identical age ${ }^{6}$. In this study, the majority (63.8\%) were postmenopausal and the mean age of menopause was 48.1 years.

Symptoms of BC vary from a painless breast lump to skin dimpling, blood stained nipple discharge and nipple retraction. Seltzer $\mathrm{MH}$, Newark found that $50 \%$ of subjects present with a new lump or a mass as the most common symptom ${ }^{7}$. Commonest clinical presentation of the index study was a palpable mass, either in isolation $(63 \%)$ or with some other associated symptoms (27.7\%). There is no national mammographic breast-screening program in Sri Lanka and the current guideline is the clinical and self-breast examination according to National Cancer Control Program based on WHO recommendation. However $7 \%$ of study population sought mammography without any symptoms. Presentation for mammography from the time of onset of symptoms could be delayed due to patient factors such as time taken for consultation or other factors related to accessibility for mammography. Majority $(63.1 \%)$ of our subjects had presented within eleven weeks with a median delay of 28 days. A German study showed that the median patient delay is 16 days and $18 \%$ of BC patients wait for more than three months prior to consultation ${ }^{22}$. 
There is strong evidence that dense breasts with a larger proportion of glandular tissue carry a higher risk of developing $\mathrm{BC}$, which has also been proven by Wolfe ${ }^{10,11,12}$. Similar association was shown in 2007, in Mayo clinic where they found that overall mammographic density is a general marker of $\mathrm{BC}$ risk but it is not specific for side or the location of the cancer developed eventually ${ }^{10}$. This concept was not supported in our study as majority $(70.5 \%)$ had involuting type P1 pattern. Dense breasts, P2 and DY types, were seen only in a minority being $4.7 \%$ and $2.9 \%$ respectively. Moreover, both pre and postmenopausal groups had involuting P1 type in the majority. This was on par with the results of a study done in India, where there was no added risk fromincreased breast density in postmenopausal women ${ }^{11}$. However, they found that there was an increased risk of developing $\mathrm{BC}$ in younger women ${ }^{11}$. This study did not reveal a higher incidence of $\mathrm{BC}$ in dense breasts even in the premenopausal age group.

A high-density mass with a spiculated margin is considered the hallmark mammographic manifestation and a predictor of invasive carcinoma. In our study $86.4 \%$ had a mass lesion on mammograms. This was relatively higher in proportion compared to a Spanish study $(74 \%)$ but it is comparable with that of the UK $(82.5 \%)^{13,14}$. Of the masses, $43.8 \%$ were in isolation without microcalcification, nipple retraction, skin thickening or architectural distortion. $67.4 \%$ of the subjects of our study had highdensity and it was comparable with a study done in USA in which $70.2 \%$ had highdensity masses ${ }^{23}$. The UK study however did SLJR Volume 2 Issue Nov 2016 not favour this finding, as they could not find a difference of density between invasive lobular carcinoma (ILC) and IDC subjects ${ }^{14}$. Spiculated margin of a mass is more commonly described in IDC than ILC. In this study, margin of the masses werespiculated in majority $(87 \%)$, but this was not supported in a Chinese study where they found $57.1 \%$ of masses to have nonspiculated margins ${ }^{5}$. The UK study found that both ILC and IDC have spiculated margins in equal number of subjects ${ }^{14}$. $27.6 \%$ of the subjects had microcalcification (MC) on the mammogram and among them $6.1 \%$ did not accompany a mass. Few studies done in Canada and in the UK found a relatively higher proportion of $\mathrm{MC}$ amounting to $40.9 \%$ and $46 \%$ respectively $^{14,15}$. BC was commonly seen as unifocal disease. However, bilateral, multifocal and multicentric disease do exist either synchronous or metachronous. Similar to other studies most (91.3\%) of our patients also presented with unifocal disease ${ }^{14}$. Upper outer quadrant predilection was observed, which contributed to $67 \%$ of $\mathrm{BC}$ among all sites. There was slight right breast predominance, but the bilateral disease was rare $(1.4 \%)$ in our sample. Invasive lobular carcinomas are known to produce bilateral disease than IDC $^{24}$. However, a few studies are on the contrary reporting invasive duct carcinoma as the most common histological type in bilateral $\mathrm{BC}^{25,26}$. Multifocal and multicentric disease was not a common finding in this study.

Size of the BC is most accurately measured at the time of histopathological diagnosis and it carries a high prognostic implication, thus used in the $\mathrm{T}$ staging. Among the 
subjects of our study, T Stage I or less than 2 $\mathrm{cm}$ tumour was found only in $10.5 \%$ of the study population. $84.3 \%$ of the masses were between $2 \mathrm{~cm}$ to $5 \mathrm{~cm}$ and were of $\mathrm{T}$ stage II. Only a minority (5.2\%) had $\mathrm{T}$ stage III disease with more than $5 \mathrm{~cm}$ mass size. A study conducted in Canada revealed that the mean tumour size was $2.5 \mathrm{~cm}^{15}$. An analysis of a BC series in USA showed that $61 \%$ presented with less than $2 \mathrm{~cm}$ tumour size ${ }^{21}$. Nevertheless, tumour size on mammography did not show any strong correlation with duration of symptoms in our study. Similarly, age of the patient and tumour size did not show significant correlation.

BIRADS 5 category carries more than $95 \%$ likelihood of a mammographic mass being malignant ${ }^{17}$. In our study, majority $(78.4 \%)$ were in BIRADS category 5 and about $5.1 \%$ of our subjects were in BIRADS 3 category.

Axillary lymph node status is still considered an important prognostic factor for BC survival despite marked evolution of breast cancer assessment by means of molecular and genetic characterization. In a few studies done in USA, it has been shown that $33 \%-36 \%$ of invasive duct carcinoma of breast has axillary LN metastases ${ }^{21,27}$. Our study showed a substantial but slightly lower frequency $(28.8 \%)$ oflocoregional LN metastases on mammography.

Conclusions:Mean age of presentationof patients with IDC was 52.2 years, which is comparable to other Asian countries but relatively lower than that of the west. Majority of the patients presented with a palpable mass, within four weeks of the onset of symptoms. Unifocal and unilateral SLJR Volume 2 Issue Nov 2016 high-density mass of $\mathrm{T}$ stage II was the commonest mammographic abnormality and size of the mass did not show a significant correlation with the duration of symptoms. Interestingly, poor correlation was found between higher mammographic breast density and IDC, among both pre and postmenopausal women, which is different from many other studies.

Acknowledgements:We thank the oncology and general surgical colleagues at Teaching Hospitals Kandy and Peradeniya respectively, for their valuable contributions.

Conflict of interest:Authors declare that they do not have any conflict of interest regarding this publication.

\section{References}

1. McPherson K, Steel C, Dixon JM. ABC of breast diseases. Breast cancer-epidemiology, risk factors and genetics. BMJ: British Medical Journal. 1994; 309(6960): 1003.

2. Curado MP. Breast cancer in the world: incidence and mortality. Saludpública de México. 2011; 53(5):372-84.

3. health.gov.lk [Internet]. Sri Lanka: National Cancer Research Program; 2010. Available from: http://www.nccp.health.gov.lk/images /PDF_PUBLICATIONS/Cancer_Incidence_ Data_2010.pdf

4. Howlader N, Noone AM, Krapcho M, Garshell J, Miller D, Altekruse SF, Kosary CL, Yu M, Ruhl J, Tatalovich Z, Mariotto A. SEER Cancer Statistics Review [Internet]. Bethesda (MD) National Cancer Institute;1975-2010. Available from: https://seer.cancer.gov/archive/csr/1975_201 2/ 
5. Jiang L, Ma T, Moran MS, Kong X, Li X, Haffty BG, Yang Q. Mammographic features are associated with clinicopathological characteristics in invasive breast cancer. Anticancer research. 2011; 31(6):2327-34.

6. Collaborative Group on Hormonal Factors in Breast Cancer. Menarche, menopause, and breast cancer risk: individual participant meta-analysis, including 118964 women with breast cancer from 117 epidemiological studies. The lancet oncology. 2012; 13(11):1141-51.

7. Seltzer MH. The significance of breast complaints as correlated with age and breast cancer. The American surgeon. 1992; 58(7):413-7.

8. Singh D, Malila N, Pokhrel A, Anttila A. Association of symptoms and breast cancer in population- based mammography screening in Finland. International journal of cancer. 2015; 136(6):E630-7.

9. Ferrini R, Mannino E, Ramsdell E, Hill L. Screening mammography for breast cancer: American College of Preventive Medicine practice policy statement. American journal of preventive medicine. 1996; 12(5):340-1.

10. Celine M Vachon, Kathleen K Brandt, Karthik Ghosh, Christopher G Scott, Shaun D Maloney, Michael J Carston, V shanePankratz, Thomas A Sellers. Mammographic breast density as a general marker of breast cancer risk. Cancer Epidemiology and Prevention Biomarkers. 2007; 16(1):43-9.

11. Attam A, Kaur N, Saha S, Bhargava SK. Mammographic density as a risk factor for breast cancer in a low risk population. Indian journal of cancer. 2008; 45(2):50.

12. Wolfe JN. Risk for breast cancer development determined by mammographic parenchymal pattern. Cancer. 1976; 37(5):2486-92.

13. Ildefonso C, Vazquez J, Guinea O, Perez A, Fernandez A, Corte MD, Junquera S, Gonzalez LO, Pravia P, Garcia-Moran M, Vizoso FJ. The mammographic appearance of breast carcinomas of invasive ductal type: relationship with clinicopathological parameters, biological features and prognosis. European Journal of Obstetrics \&Gynecology and Reproductive Biology. 2008;136(2):224-31.

14. Cornford EJ, Wilson AR, Athanassiou E, Galea M, Ellis IO, Elston CW, Blamey RW. Mammographic features of invasive lobular and invasive ductal carcinoma of the breast: a comparative analysis. The British journal of radiology. 1995;68(809):450-3.

15. Naseem M, Murray J, Hilton JF, Karamchandani J, Muradali D, Faragalla H, Polenz C, Han D, Bell DC, Brezden-Masley C. Mammographic microcalcifications and breast cancer tumorigenesis: a radiologicpathologic analysis. BMC cancer. 2015; 15(1):307.

16. Harris JR, Lippman ME, Veronesi U, Willett W. Breast cancer. New England Journal of Medicine. 1992; 327(6):390-8.

17. BI-RADS Atlas, Mammography. American College of Radiology. $5^{\text {th }}$ edition; 2013.

18. Sinn HP, Kreipe H. A brief overview of the WHO classification of breast tumors. Breast Care. 2013; 8(2):149-54.

19. Malhotra GK, Zhao X, Band H, Band V. Histological, molecular and functional subtypes of breast cancers. Cancer biology \& therapy. 2010; 10(10):955-60.

20. American Cancer Society. Breast cancer facts \& figures 2011-2012. American Cancer Society INC. 2011; 1(34).

21. Li CI, Uribe DJ, Daling JR. Clinical characteristics of different histologic types of breast cancer. British journal of cancer. 2005 Oct 31;93(9):1046-52.

22. Arndt V, Stürmer T, Stegmaier C, Ziegler H, Dhom G, Brenner H. Patient delay and stage of diagnosis among breast cancer patients in Germany-a population based study. British journal of cancer. 2002; 86(7):1034-40.

23. Woods RW, Sisney GS, Salkowski LR, Shinki K, Lin Y, Burnside ES. The mammographic density of a mass is a significant predictor of breast cancer. Radiology. 2011; 258(2):417-25. 
24. GraziaArpino, Valeria J Bardou, Gary M Clark, Richard M Elledge. Infiltrating lobular carcinoma of the breast; tumour characteristics and clinical outcome. Breast Cancer Research 2004; 6: 149-156

25. Soo Jung Gong, Sun Young Rha, HeiCheulJeung, Jae Kyung Roh, Woo Ick Yang, Hyun Cheol Chung, Bilateral breast cancer; Differentildiagnois using histological and biological parameters.JapaneseJounal of Clinical Oncology 2007; 37(7): 487-492
26. Kadioğlu H, Özbaş S, Akcan A, Soyder A, Soylu L, Koçak S, Cantürk NZ, Tükenmez $\mathrm{M}$, Müslümanoğlu M. Comparison of the histopathology and prognosis of bilateral versus unilateral multifocal multicentric breast cancers. World journal of surgical oncology. 2014; 12(1):266.

27. Silverstein MJ, Skinner KA, Lomis TJ. Predicting axillary nodal positivity in 2282 patients with breast carcinoma. World journal of surgery. 2001; 25(6):767-7

Table 1. Demographic and clinical characteristics of the IDC subjects

\begin{tabular}{lrr}
\hline Variables & Frequency (\%) & Mean/Median \\
\hline Histological type (n=209) & $168(80.4)$ & \\
IDC & $9(4.3)$ \\
IDC+DCIS & $22(10.5)$ \\
DCIS & $10(4.8)$ & \\
Other & & Mean age 52.2(SD \pm 1.1$)$ \\
Age (n=177) & $19(10.7)$ & \\
$30-39$ & $57(32.2)$ \\
40-49 & $55(31.1)$ \\
$50-59$ & $36(20.4)$ \\
60-69 & $10(5.6)$ \\
>70 & & \\
Menopausal status (n=174) & $63(36.2)$ \\
Pre & $111(63.8)$ \\
Post & & \\
Clinical Presentation (n=173) & $109(63.0)$ \\
Mass(in isolation) & $4(2.3)$ \\
Mastalgia & $39(22.5)$ \\
Mass+mastalgia & $4(2.3)$ \\
Mass+mastalgia+nipple discharge & $1(0.6)$ \\
Mass+mastalgia+skin infiltration & $1(0.6)$ \\
Mass+nipple discharge & $3(1.7)$ \\
Mass+skin infiltration & $12(7.0)$ \\
Asymptomatic & & \\
Duration of Symptoms (n=149) & $23(15.4)$ \\
$<1 \mathrm{w}$ & $46(30.9)$ \\
1-3w & $48(32.2)$ \\
4-11w & $19(12.8)$ \\
12-24w & $13(8.7)$ \\
=>25w &
\end{tabular}


Table 2. Correlation between menopausal status with Wolfe breast density grades

\begin{tabular}{|l|r|r|r|r|r|}
\hline & \multicolumn{5}{|c|}{ Wolfe Breast Density type (\%) } \\
\cline { 2 - 7 } & $\mathrm{N} 1$ & $\mathrm{P} 1$ & $\mathrm{P} 2$ & $\mathrm{DY}$ & Total \\
\cline { 2 - 7 } & & & & & \\
\hline
\end{tabular}

Missing information-7; Spearman correlation coefficient $-0.07, P=0.001$

Table 3. Mammographic Characteristics of IDC

\begin{tabular}{lr}
\hline Variables & Frequency $(\boldsymbol{\%})$ \\
\hline Mass (n=153) & $67(43.8)$ \\
Mass in isolation & $86(56.2)$ \\
Mass+ (Skin thickening [ST]+Architecture distortion & \\
[AD]+Microcalcification [MC]) & \\
Site of the mass (n=112) & $49(43.8)$ \\
RUOQ & $9(8.0)$ \\
RUIQ & $26(23.2)$ \\
LUOQ & $11(9.8)$ \\
LUIQ & $1 \quad(0.9)$ \\
RLOQ & $7(6.3)$ \\
RLIQ & $6(5.3)$ \\
LLOQ & $3(2.7)$ \\
LLIQ & \\
Position of the mass $(\mathbf{n}=\mathbf{1 4 2})$ & $140(98.6)$ \\
Unilateral & $2 \quad(1.4)$ \\
Bilateral & \\
Focus of the mass $(\mathbf{n}=\mathbf{1 3 8})$ & $126(91.3)$ \\
Unifocal & $8(5.8)$ \\
Multifocal & $4(2.9)$ \\
Multicentric & \\
Mass Density $(\mathbf{n = 1 3 8 )}$ &
\end{tabular}




\begin{tabular}{|c|c|}
\hline High density & $93 \quad(67.4)$ \\
\hline Isodense & $37 \quad(26.8)$ \\
\hline Other & $8 \quad(5.8)$ \\
\hline \multicolumn{2}{|l|}{ Margin of the mass $(n=146)$} \\
\hline Spiculated & $127 \quad(87.0)$ \\
\hline Non spiculated & 19 (13.0) \\
\hline \multicolumn{2}{|l|}{ Size of the mass and T stage $(n=153)$} \\
\hline$<2 \mathrm{~cm}(\mathrm{~T} 1)$ & $16(10.5)$ \\
\hline $2-5 \mathrm{~cm}(\mathrm{~T} 2)$ & $129(84.3)$ \\
\hline$>5 \mathrm{~cm}(\mathrm{~T} 3)$ & $8(5.2)$ \\
\hline \multicolumn{2}{|l|}{ Architectural distortion (AD) $(n=14)$} \\
\hline $\mathrm{AD}$ in isolation & $11(78.6)$ \\
\hline $\mathrm{AD}+\mathrm{ST}$ & $3(21.4)$ \\
\hline \multicolumn{2}{|l|}{ Microcalcification (MC) $(\mathrm{n}=49)$} \\
\hline $\mathrm{MC}+$ Mass $+\mathrm{AD}+\mathrm{ST}+$ Nipple retraction & $46(93.9)$ \\
\hline $\mathrm{MC}+\mathrm{AD}+\mathrm{ST}$ & $3 \quad(6.1)$ \\
\hline \multicolumn{2}{|l|}{ BIRADS category $(n=158)$} \\
\hline 3 & $(5.1)$ \\
\hline 4 & 18 (11.4) \\
\hline 5 & $124(78.4)$ \\
\hline 6 & $8(5.1)$ \\
\hline
\end{tabular}

Table 4. Correlation between the duration of symptoms and size of the mass

\begin{tabular}{|c|c|c|c|c|c|}
\hline & \multicolumn{3}{|c|}{ Size of the Mass $(\mathrm{cm})$} & \multirow{2}{*}{ Total } \\
\hline & & $<2$ & $2-5$ & $>5$ & \\
\hline \multirow{5}{*}{$\begin{array}{l}\text { Duration of } \\
\text { presentation }\end{array}$} & $<\mathbf{1 w}$ & 3 & 10 & 2 & 15 \\
\hline & $1-3 w$ & 4 & 40 & 2 & 46 \\
\hline & 4-11w & 6 & 37 & 3 & 46 \\
\hline & $12-24 w$ & 2 & 17 & 0 & 19 \\
\hline & $=>25 w$ & 0 & 10 & 1 & 11 \\
\hline \multicolumn{2}{|l|}{ Total } & 15 & 114 & 8 & 137 \\
\hline
\end{tabular}


Table 5. Correlation between the age and size of the mass

\begin{tabular}{|l|r|r|r|r|r|r|r|}
\hline \multicolumn{2}{|c|}{} & \multicolumn{5}{|c|}{ Age group } & \\
\cline { 2 - 8 } \multicolumn{2}{|c|}{} & $\mathbf{3 0 - 3 9}$ & $\mathbf{4 0 - 4 9}$ & $\mathbf{5 0 - 5 9}$ & $\mathbf{6 0 - 6 9}$ & $>\mathbf{6 9}$ & Total \\
\hline Mass Size & $<\mathbf{2 c m}$ & 1 & 3 & 8 & 4 & 0 & 16 \\
\cline { 2 - 8 } & $\mathbf{2 - 5 c m}$ & 15 & 39 & 38 & 28 & 9 & 129 \\
\cline { 2 - 8 } & $>\mathbf{5 c m}$ & 0 & 3 & 2 & 3 & 0 & 8 \\
\hline Total & 16 & 45 & 48 & 35 & 9 & 153 \\
\hline
\end{tabular}

Missing information - 24; Spearman correlation - 0.07; P- 0.57

Table 6. Axillary LN status on mammography and histopathology

\begin{tabular}{|l|l|l|l|}
\hline & Histology + & Histology - & Total \\
\hline Mammo + & $21(41.2 \%)$ & $28(22.2 \%)$ & 49 \\
\hline Mammo - & $30(58.8 \%)$ & $98(77.8 \%)$ & 128 \\
\hline Total & $\mathbf{5 1 ( 1 0 0 . 0 \% )}$ & $\mathbf{1 2 6}(\mathbf{1 0 0 . 0 \% )}$ & \\
\hline
\end{tabular}

Sensitivity of mammography $=41.2 \%$

Specificity of mammography $=77.8 \%$ 